\title{
Activation of SGK1.1 Upregulates the M-current in the Presence of Epilepsy Mutations
}

\author{
Elva Martin-Batista', Rían W. Manville², Belinda Rivero-Pérez ${ }^{1}$, \\ David Bartolomé-Martín ${ }^{1}$, Diego Alvarez de la Rosa', Geoffrey W. Abbott ${ }^{2}$ and \\ Teresa Giraldez ${ }^{1 *}$
}

1 Departamento de Ciencias Medicas Basicas and Instituto de Tecnologias Biomedicas, Universidad de La Laguna, San Cristóbal de La Laguna, Spain, ${ }^{2}$ Bioelectricity Laboratory, Department of Physiology and Biophysics, School of Medicine, University of California, Irvine, Irvine, CA, United States

In the central nervous system, the M-current plays a critical role in regulating subthreshold electrical excitability of neurons, determining their firing properties and responsiveness to synaptic input. The M-channel is mainly formed by subunits Kv7.2 and Kv7.3 that co-assemble to form a heterotetrametric channel. Mutations in Kv7.2 and Kv7.3 are associated with hyperexcitability phenotypes including benign familial neonatal epilepsy (BFNE) and neonatal epileptic encephalopathy (NEE). SGK1.1, the neuronal isoform of the serum and glucocorticoids-regulated kinase 1 (SGK1), increases M-current density in neurons, leading to reduced excitability and protection against seizures. Herein, using two-electrode voltage clamp on Xenopus laevis oocytes, we demonstrate that SGK1.1 selectively activates heteromeric Kv7 subunit combinations underlying the M-current. Importantly, activated SGK1.1 increases M-channel activity in the presence of two different epilepsy mutations found in Kv7.2, R207W and A306T. In addition, proximity ligation assays in the $\mathrm{N} 2 \mathrm{a}$ cell line allowed us to address the effect of these mutations on Kv7-SGK1.1-Nedd4 molecular associations, a proposed pathway underlying augmentation of M-channel activity by SGK1.1

Keywords: serum and glucocorticoid-induced kinase, Kv7 channels, KCNQ, epilepsy, SGK1.1

\section{INTRODUCTION}

Mutations affecting ion channel subunit-encoding genes underly several forms of epilepsy (for a recent review see Oyrer et al., 2018). In the CNS, KCNQ2, and KCNQ3 genes encode, respectively, the Kv7.2 and $\mathrm{Kv7.3} \mathrm{K}^{+}$channel subunits, which co-assemble and form a heterotetrametric channel underlying the M-current (Brown and Adams, 1980; Wang et al., 1998). Kv7.4 and Kv7.5 also contribute to generating the M-current in the CNS, although to a lesser extent (Kubisch et al., 1999; Lerche et al., 2000; Schroeder et al., 2000; Shah et al., 2002). M-channels are active at subthreshold membrane potentials (near $-60 \mathrm{mV}$ ). Their activation is slow and thus they do not contribute to the repolarization of individual action potentials. M-channels do not inactivate and therefore generate a steady voltage-dependent outward current that leads to the stabilization of membrane potential, contributing to setting the resting membrane potential (RMP). The M-current is negatively regulated by activation of muscarinic acetylcholine receptors through phosphatidylinositol 4,5-bisphosphate $\left(\mathrm{PIP}_{2}\right)$ depletion. Thus, the M-current plays a critical role in dynamically regulating subthreshold electrical excitability of neurons, determining their firing properties and responsiveness to synaptic input (Brown and Adams, 1980; 
Constanti and Brown, 1981; Wang and McKinnon, 1995). Mutations in KCNQ2 and KCNQ3 associate with hyperexcitability phenotypes, including benign familial neonatal epilepsy (BFNE) and neonatal epileptic encephalopathy (NEE) (Singh et al., 1998, 2003; Soldovieri et al., 2014; Nappi et al., 2020). BFNE is a dominantly inherited condition affecting newborns and characterized by the occurrence of focal, multifocal or generalized seizures. BFNE patients have a higher risk of developing epilepsy later in life when compared to the general population (Ronen et al., 1993; Plouin and Anderson, 2005). In NEE, encephalopathy is present from birth and persists during a period when seizures are uncontrolled, leading to developmental impairment. Cessation of seizures generally occurs between age 9 months and 4 years (Miceli et al., 2010). Modulation of M-current constitutes a potential target to treat epilepsy and other diseases driven by neuronal hyperexcitability such as neuropathic pain, ischemia and schizophrenia (Wulff et al., 2009). In fact, a number of pharmacological tools have been identified which modulate M-current. Among them, retigabine and flupirtine have demonstrated ability to prevent seizure activity both in animal models and in clinical trials (Wickenden et al., 2004). However, due to drug-induced liver injury and tissue discoloration by flupirtine and retigabine, respectively, both drugs were recently discontinued (Michel et al., 2012; Clark et al., 2015; Surur et al., 2019). Since retigabine and flupirtine adverse effects are not related to their mechanism of action, it seems promising to continue studying M-current modulators and activation pathways as effective and clinically relevant anticonvulsant treatments (Surur et al., 2019). We previously described that SGK1.1, the neuronal isoform of the serum and glucocorticoid-induced kinase 1 (SGK1), increases membrane abundance of Kv7.2/3 channels in a PIP 2 -dependent manner, resulting in augmented current levels and providing a convergence point to $\mathrm{M}$-channel regulation (Miranda et al., 2013). Recently, we described that augmentation of M-channel activity by transgenic co-expression of constitutively active SGK1.1 potently reduces seizure severity and duration in a kainic acid mouse model of temporal lobe epilepsy (Armas-Capote et al., 2020) and contributes to neuroprotection (Martin-Batista et al., 2021). However, the wider effects of SGK1.1 on the Kv7 family of channels forming the neuronal M-current have not been directly addressed and, most importantly, its potential role in rescuing epileptogenic mutations such as those found in Kv7.2 (R207W and A306T) is currently unknown. R207W is a mutation that neutralizes a charged amino-acid residue within the S4 transmembrane segment of the Kv7.2 voltage sensor domain, slowing voltage-sensor dependent channel activation and resulting in BFNE (Dedek et al., 2001). Mutation A306T is located in the S6 segment of Kv7.2, within the pore domain. This mutant exhibits reduced currents but retains many of the biophysical characteristics of the wild-type channel (Xiong et al., 2008). In this work, we examined the regulation by SGK1.1 of several combinations of Kv7 subunits, providing evidence that SGK1.1 regulates not only Kv7.2/3, but also Kv7.4/5, M-current forming heteromeric channels. Fully activated SGK1.1 was able to partially restore activity in Kv7.2/3 channels harboring loss-offunction Kv7.2 epileptogenic mutations, further supporting its role as a possible avenue to counteract neuronal hyperexcitability by potentiating the M-current.

\section{MATERIALS AND METHODS}

Animal handling and experimental procedures were approved by Universidad de La Laguna Ethics Committee and conform to Spanish and European guidelines for protection of experimental animals (RD53/2013; 2010/63/EU).

\section{Plasmid Constructs, cRNA Synthesis and Oocyte Microinjection}

pSRC5 plasmids carrying human Kv7.2 and Kv7.3 were kindly provided by Dr. Alvaro Villarroel (CSIC Institute of Biofisika, Vizcaya, Spain). pTLN plasmids carrying human KCNQ1, Kv7.3(A315T) and KCNQ4-5 as well as KCNE1 cloned into pRAT have been previously described (Manville et al., 2018). Mouse SGK1.1 (wild type and constitutively active mutant S515D) cloned into pcDNA3.1/V5-His-TOPO (Invitrogen) were kind gifts from Dr. Cecilia Canessa (Yale University, New Haven, CT, United States). Kinase-dead mutant K220A was described previously (Wesch et al., 2010). SGK1.1 constructs were cloned into pECFP-N1 and pEYFP-N1 (Clontech) plasmids for expression in mammalian cells (Wesch et al., 2010). pcDNA3.1 containing N-methyl-D-aspartate receptor (NMDAR) subunit 1 (NR1) fused to YFP was a gift from Dr. Stefano Vicini (Addgene plasmid \#17928; Luo et al., 2002). KCNQ2 mutants were generated by site-directed mutagenesis using a QuikChange kit according to manufacturer's protocol (Stratagene, San Diego, CA, United States). cRNA transcripts encoding human KCNQ15 were generated by in vitro transcription using the T7 polymerase mMessage mMachine kit (Thermo Fisher Scientific) or SP6 polymerase mMessage mMachine kit (Thermo Fisher Scientific), after vector linearization. cRNA was quantified by spectrophotometry. Oocytes were obtained from Ecocyte Bioscience (Austin, TX, United States) or harvested from Xenopus laevis females and cRNAs microinjected as previously described (Miranda et al., 2013; Manville et al., 2018). Briefly, defolliculated stage $\mathrm{V}$ and VI oocytes were injected with Kv7 channel $\alpha$ subunit cRNAs (10 ng), alone or with SGK1.1 cRNA (10 ng). Oocytes were incubated at $16^{\circ} \mathrm{C}$ in ND96 solution (in mM: $96 \mathrm{NaCl}, 2 \mathrm{KCl}, 1.8 \mathrm{CaCl}_{2}, 1 \mathrm{MgCl}_{2}, 20$ HEPES, pH 7.6) containing gentamycin with daily washing for 1-2 days prior to electrophysiological recordings.

\section{Two-Electrode Voltage Clamp}

Two electrode voltage clamp (TEVC) was performed at room temperature with an OC-725C amplifier (Warner Instruments, Hamden, CT, United States) and pClamp8 software (Molecular Devices, Sunnyvale, CA, United States) 1-2 days after cRNA injection as described above. Oocytes were placed in a small volume oocyte bath in recording solution (in mM: $96 \mathrm{NaCl}, 4$ $\mathrm{KCl}, 1 \mathrm{MgCl}_{2}, 1 \mathrm{CaCl}_{2}, 10 \mathrm{HEPES}, \mathrm{pH}$ 7.6) under a dissection microscope. Pipette resistance was 1-5 M $\Omega$ when filled with $3 \mathrm{M}$ $\mathrm{KCl}$. Currents were recorded in response to pulses between -80 $\mathrm{mV}$ and $+40 \mathrm{mV}$ at $20 \mathrm{mV}$ intervals from a holding potential 
of $-70 \mathrm{mV}$, followed by a $-30 \mathrm{mV}$ pulse to yield current-voltage relationships, current magnitude and for quantifying activation rate. TEVC data analysis was performed with Clampfit 10.6 (Molecular Devices). Half-maximal activation voltage calculated from Boltzmann equation fits to activation curves and activation constants were calculated from monoexponential fits to evoked currents, as described (Miranda et al., 2013).

\section{Cell Culture, Transfection and Genome Editing}

Mouse neuroblastoma Neuro2A (N2a) cells were obtained from American Type Culture Collection (Manassas, VA) and maintained in DMEM supplemented with 10\% FBS. Cells were transfected 24-48 h before the experiment using Jetprime (Polyplus Transfection) following the manufacturer's instructions. Sgk1 knockout in N2a cells was performed using CRISPR/Cas9 induced non-homologous end joining using one plasmid system. SpCas9 and trans-activating CRISPR RNA (tracrRNA) were expressed using the pX459 plasmid (a gift from Dr. Feng Zhang, Addgene plasmid \#62988, Ran et al., 2013), which includes a puromycin resistance cassette. Two guide RNAs (gRNA) were designed to cut exons 6 and 8 simultaneously, using the Breaking-Cas tool (Oliveros et al., 2016) and cloned into pX459 using the BbsI site. These exons were selected because they include the catalytic domain common to all isoforms of the kinase (Arteaga et al., 2008), ensuring knockout of both isoforms. Thirty-six hours after transfection, $3 \mu \mathrm{g} / \mathrm{ml}$ puromycin was added, and after $48 \mathrm{~h}$, surviving cells were split and seeded at low density in $10 \mathrm{~cm}$ dishes. Single cell clones were collected using cloning cylinders and split in multi-well plates. PCR screening was performed using primers flanking exons 6-8, which produce a $681 \mathrm{bp}$ amplicon from the wild type gene (F-check mSGK1: 5' GTAAGGCTGTGTGCAGCGTA $3^{\prime}$ and R-check mSGK1: 5' TCAAACCCAAACCAAGCAAT 3'). Clones that showed amplicon absence or altered size were selected to perform western blot analysis of SGK1 expression. Thus, the absence of SGK1 and SGK1.1 was confirmed, selecting an $\mathrm{N} 2 \mathrm{a}$ clone knockout.

\section{Antibodies}

For SGK1.1 detection we used a rabbit polyclonal antibody produced in our laboratory (Martin-Batista et al., 2021). SGK1 was detected using rabbit anti-SGK1 polyclonal antibody (Abcam, ab43606). Kv7.2 was detected with rabbit anti-Kv7.2 polyclonal antibody (Abcam, ab22897). YFP-tagged SGK1.1 and GFP-tagged Kv7 subunits were detected using mouse anti-GFP monoclonal antibody (Abcam, ab290). Nedd4-2 was detected with a rabbit polyclonal antibody from Cell Signaling (4013S). GAPDH was detected with a mouse monoclonal antibody (Abcam, 9484). Secondary antibodies conjugated to fluorophores were obtained from Thermo Fisher Scientific (Alexa 594, A11042; Alexa-488, A-11008).

\section{Western Blotting}

$\mathrm{N} 2 \mathrm{a}$ cells or pools of oocytes injected with the same cRNA combinations were lysed in TENT buffer (in mM: 50 Tris- $\mathrm{HCl}, 5$
EDTA, $150 \mathrm{NaCl}, 1 \%$ Triton X-100, pH 7.4) containing protease and phosphatase inhibitors (Roche). Centrifugation supernatants were collected, mixed with Laemmli loading buffer, and heated up to $95^{\circ} \mathrm{C}$ for $5 \mathrm{~min}$. Proteins were separated in $10 \%$ polyacrylamide gels (Mini- PROTEAN TGX Stain-Free Gels, Bio-Rad) and transferred to polyvinyl difluoride membranes (Bio-Rad) for blotting with anti-Kv7.2 and anti-GADPH antibodies referenced above. Chemiluminescence signals were quantified using Image Lab $^{\circledR}$ software 6.0 (Bio-Rad).

\section{Proximity Ligation Assay}

Cells grown on coverslips were transiently transfected with plasmids encoding the combinations of indicated variants of SGK1.1, Nedd4-2, Kv7.2, and Kv7.3, 24 h prior to the assay, using a JetPrime ${ }^{\circledR}$ transfection kit. After fixation with $4 \%$ formaldehyde, cells were processed for the proximity ligation assay (PLA) to detect physical proximity of different protein pairs expressed in Sgk1-KO N2a cells following the manufacturer's protocol (Duolink, Olink Biosciences) and using the antibodies indicated in each case. Cells were then mounted, and images obtained using a confocal microscope (Leica SP8), 40× magnification and a zoom factor of 2 . Images were analyzed using the software provided by the manufacturer (Duolink Image Tool). Results are expressed as average number of puncta/cell area normalized to control conditions obtained using non-interacting proteins (cells expressing NR1 and SGK1.1) and compared to our reference condition of cells only transfected with the channel subunits, Kv7.2 and Kv7.3.

\section{RESULTS}

\section{SGK1.1 Boosts Activity of Heteromeric Kv7 Channels Underlying the Neuronal M-current}

In the first set of experiments, we compared the effect of wild type (WT) SGK1.1 with that of constitutively active SGK1.1 mutant S515D on Kv7.2/3-mediated M-currents. We found that coexpression of WT SGK1.1 with Kv7.2/3 resulted in significantly increased peak and tail currents (Figures 1A-C) without altering normalized conductance (Figure 1D), as we have previously described (Miranda et al., 2013). Co-expression of SGK1.1S515D activated Kv7.2/3-mediated currents to the same extent as WT SGK1.1 (Figures 1A-C), indicating that endogenous activation of the kinase is enough to maximize its effects on the channel. Next, in order to assess whether the effect was specific on heterometric assemblies, we tested oocytes expressing heteromeric Kv7.3/5, which is a described combination associated to M-current (Schroeder et al., 2000). Our results showed that tail currents were significantly increased in presence of SGK1.1 at $0,+20$ and $+40 \mathrm{mV}$ (Figures 2A-D). Heterologous expression of Kv7.4 or Kv7.5 resulted in $\mathrm{K}^{+}$currents smaller than those elicited by heteromeric Kv7.2/3/5 (Supplementary Figure 1). In contrast to the effect observed with heteromeric channels, co-expression of SGK1.1 produced an inhibitory effect on the tail current elicited by Kv7.4 (Supplementary Figure 1C) 
whereas no alteration was observed in Kv7.5-mediated currents (Supplementary Figures 1E-H). When we co-expressed Kv7.1 and accessory subunit KCNE1, characteristic slow activating, voltage-dependent current reminiscent of the slow cardiac $\mathrm{K}^{+}$repolarizing current $\left(\mathrm{I}_{\mathrm{Ks}}\right)$ was elicted (Supplementary Figure 2A). Co-expression of SGK1.1 affected neither amplitude nor conductance (Supplementary Figures 2B-D) of the Kv7.1KCNE1 currents. Altogether, this series of experiments indicates that SGK1.1 regulates heteromeric channels Kv7.2/3 and Kv7.3/5 mediating neuronal M-currents, but not their cardiac counterpart, Kv7.1/KCNE1.

\section{Activated SGK1.1 Up-Regulates Channel Activity of Kv7.2 Epilepsy Mutants R207W and A306T in Heteromeric Assembly With Kv7.3}

We next tested the potential role of SGK1.1 in modulating function of Kv7 channels carrying epileptogenic mutations such as those described in Kv7.2 (R207W and A306T). Expression of epilepsy mutant Kv7.2 (R207W) (Figure 3A) along with Kv7.3 produced smaller currents with slower voltage-dependent activation profile [Figures 3B-E, activation constants 1,006 ms (R207W) vs. $104 \mathrm{~ms}$ (WT)], in agreement with previous reports (Dedek et al., 2001). Co-expression of wild type SGK1.1 did not produce any change in the amplitude of the R207Wmodified M-current. Strikingly, however, SGK1.1 (S515D) increased Kv7.2 (R207W)/WT Kv7.3 peak and tail currents significantly (Figures 3B-E). We continued our study by testing the mutation A306T, located within the pore domain in the S6 segment of the subunit (Figure 3A). These mutants have been shown to maintain most Kv7.2 biophysical characteristics but produce significantly reduced currents (Xiong et al., 2008). Our experiments co-expressing Kv7.2 (A306T) with WT Kv7.3 resulted in non-detectable currents (data not shown). We circumvented this limitation by using a previously described mutation (A315T) in the pore of Kv7.3 that greatly increases current amplitude (Gomez-Posada et al., 2011). We previously demonstrated that SGK1.1 does not affect currents elicited by Kv7.3 (A315T), but does increase those of the heteromeric Kv7.2/3 (A315T) channel (Miranda et al., 2013). When analyzing the currents elicited by mutant Kv7.2 (A306T) in heteromeric assembly with Kv7.3 (A315T) we observed similar findings to those with Kv7.2 (R207W). Only the expression of the SGK1.1 (S515D) mutant resulted in substantial increase of the current (Figures 4A-C), with negligible effects on voltage dependence of activation (Figure 4D). In summary, this set of results demonstrates that fully activated SGK1.1 partially restores function of Kv7.2/3 channels carrying Kv7.2 loss-offunction mutations.

\section{SGK1.1 Associates With Kv7.2/3 Channels and Nedd4-2}

To gain further information about the mechanism underlying the effects of these epileptogenic mutations, we next tested the levels of Kv7.2 protein expression in two heterologous expression systems-injected oocytes and transfected N2a cells.
Our first observation was that mutations R207W and A306T each significantly decreased the abundance of Kv7.2 protein in oocytes, which had been previously suggested but not directly tested. Additional experiments with and without co-expressing SGK1.1 showed that neither WT SGK1.1 nor activated SGK1.1 (S515D) restored the expression levels of Kv7.2 mutants to WT values (Figures 5A,B). Similar results were observed when the different constructs were expressed in N2a cells (Figures 5C,D). We previously demonstrated that SGK1.1 increases Kv7.2/3 current by augmenting channel membrane abundance through a Nedd4-2-mediated pathway (Miranda et al., 2013). Whether these proteins interact, forming stable complexes, had not been directly addressed. To this aim, we generated a mouse neuroblastoma $\mathrm{N} 2 \mathrm{a}$ cell line with genetic inactivation of all endogenous $S g k 1$ isoforms, in which we performed experiments using the proximity ligation assay (PLA) (Figure 5E). Expression of the transfected constructs and the absence of endogenous SGK1.1 in this genetically modified N2a cell line were confirmed by immunocytochemistry (Supplementary Figure 3). As shown in Figures 5F,G, PLA signals were significantly more numerous in cells expressing Kv7.2 or Kv7.3 along with activated SGK1.1 (S515D) compared to the reference control only expressing Kv7.2/3. Our data additionally demonstrate that co-expression of heteromeric Kv7.2/3 significantly increased the association of activated SGK1.1 to the channel compared to homomeric Kv7.3. These differences in signal strength between homomeric and heteromeric structures suggest that association of SGK1.1 (S515D) with the channel becomes facilitated in the heteromeric configuration, which supports previous electrophysiological data showing that the SGK1.1 effect requires the heteromeric assembly of Kv7.2/3 channels (Miranda et al., 2013). We then tested whether the catalytic activity of SGK1.1 was required for this association by introducing the mutation K220A in the ATPbinding cassette, which abolishes the kinase activity (Wesch et al., 2010). In this case, PLA signals were significantly reduced, indicating that the association between the kinase and the heteromeric channel was significantly impaired. A logical question following these findings was whether epilepsy mutations in Kv7.2 may disrupt the association between the kinase and channels. Interestingly, our results showed detectable signals demonstrating molecular proximity between the kinase and the heteromeric channels containing epileptogenic mutations (Figures 5F,G), even at the lower expression levels of these mutants revealed in our study (Figures 5A,B). In summary, our data demonstrate that activated SGK1.1 forms close associations with Kv7 channels, preferably in heteromeric combinations, even in the presence of loss-of-function mutations. Consistent with previous functional studies, this interaction requires intact SGK1.1 kinase activity.

The ubiquitous isoform SGK1 inactivates Nedd4-2 by phosphorylating it at residue S448 (Debonneville et al., 2001). We previously demonstrated that SGK1.1 also phosphorylates Nedd4-2 (Armas-Capote et al., 2020), and that SGK1.1 upregulates Kv7.2/3 currents via a Nedd4-2-mediated mechanism (Miranda et al., 2013). In this work, we used the PLA approach to determine the existence of stable associations between Nedd4-2 and SGK1.1 (S515D). As shown in Figures 6A,B, a 

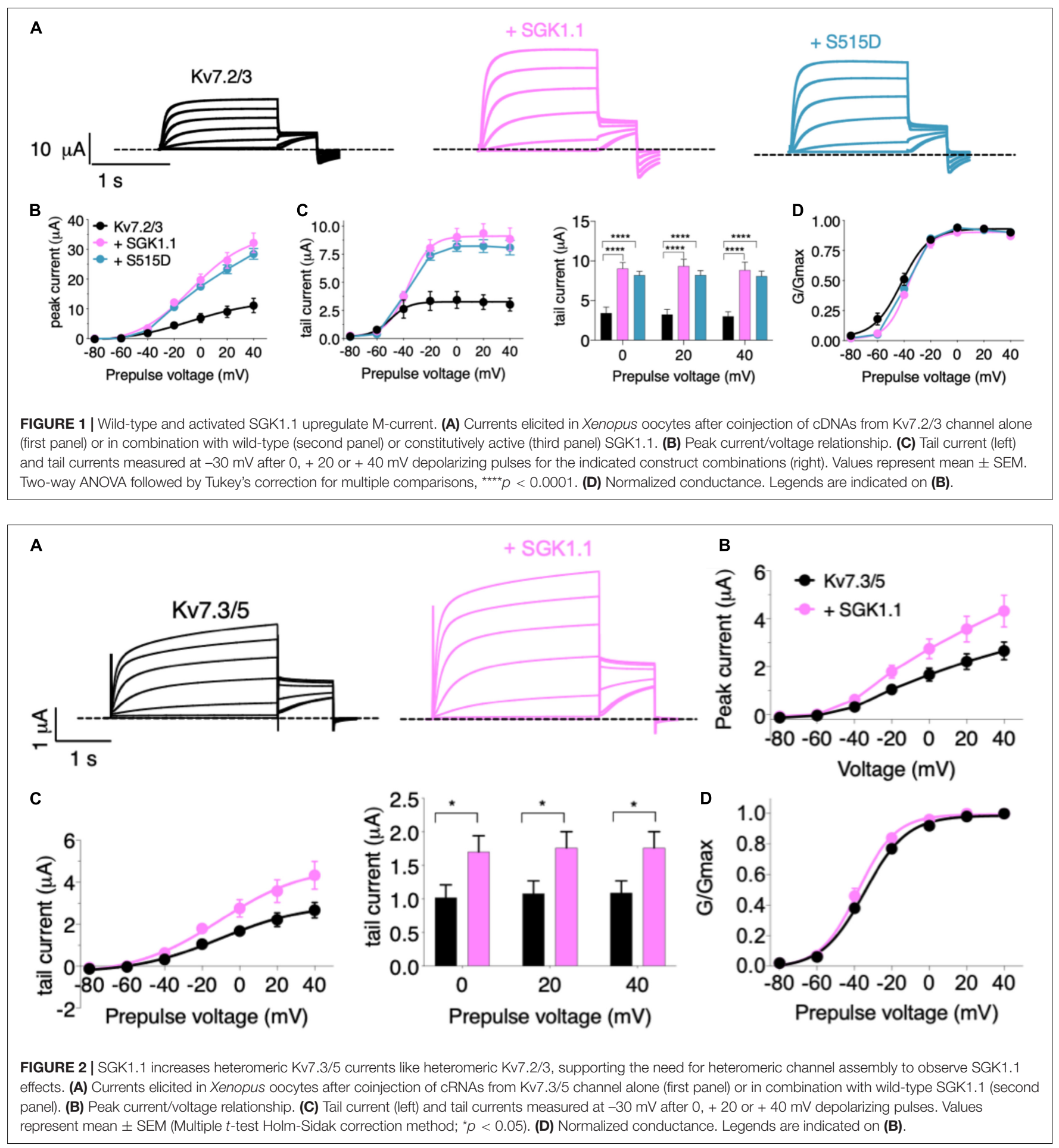

significantly increased number of PLA signals was observed when SGK1.1 (S515D) and Nedd4-2 were co-expressed, suggesting that activated SGK1.1 associates with Nedd4-2. This observation is in agreement with previous reports suggesting a direct interaction between Kv7 and Nedd4-2 (Ekberg et al., 2007). In contrast, our data demonstrated that this association is significantly reduced in the presence of the kinase dead mutant SGK1.1 (K220A), as can be inferred from the significantly reduced PLA signal levels when this protein combination was tested (Figures 6A,B). A straightforward conclusion of our data is that SGK1.1 does interact with Nedd4-2 in a phosphorylation-dependent manner.

Finally, we studied the association between Nedd4- 2 and Kv7 channels and whether SGK1.1 co-expression alters this complex. Our results in Figure 6C show significantly augmented 

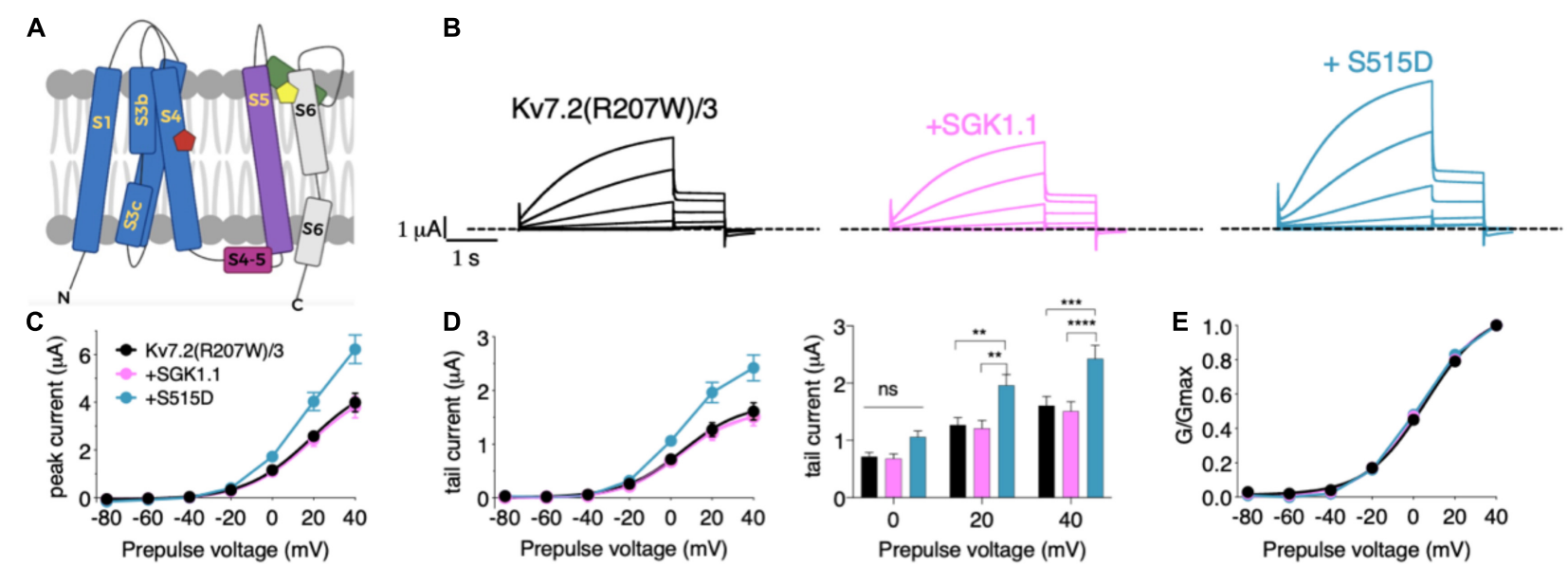

FIGURE 3 | Constitutively active form SGK1.1 (S515D) upregulates Kv7.2 epilepsy mutation R207W in heteromeric assembly with Kv7.3 while WT SGK1.1 fails to. (A) Schematic representation of Kv7 channel structure. The basic organization of potassium channels is a tetramer with each monomer containing one pore-forming domain (PD) (transmembrane segments S1-S4) and a voltage sensor domain (VSD) (transmembrane segments S5-S6). Epilepsy mutants are represented as colored pentagons (R207W in red and A306T in yellow). (B) Currents elicited in Xenopus oocytes after coinjection of cDNAs from Kv7.2 (R207W)/3 channel alone (first panel) or in combination with wild-type (second panel) or constitutively active (third panel) SGK1.1. (C) Peak current/voltage relationship. (D) Tail current (left) and tail currents measured at $-30 \mathrm{mV}$ after $0,+20$ or $+40 \mathrm{mV}$ depolarizing pulses for the indicated construct combinations (right). Values represent mean \pm SEM (Two-way ANOVA, Tukey's correction for multiple comparisons; ns, not significant; ${ }^{\star \star} p<0.01$; ${ }^{\star \star *} p<0.0005$; $\left.{ }^{\star \star \star *} p<0.0001\right)$. (E) Normalized conductance. Legends are indicated on (C).

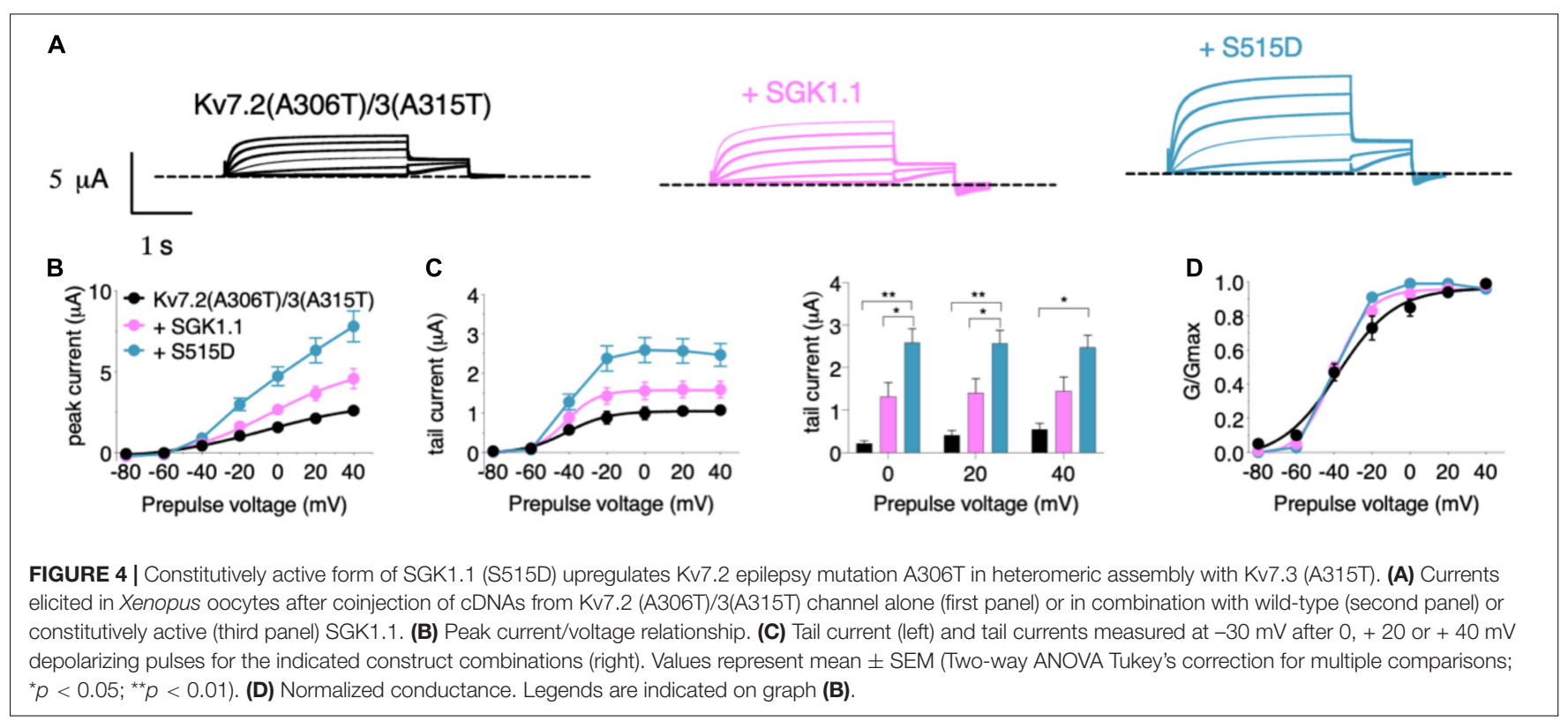

PLA signals in cells co-expressing Kv7.2/3 and Nedd4-2 as compared with the control, consistent with the association of both proteins. Strikingly, this interaction was significantly reduced when the activated form of SGK1.1 was co-expressed, but not in the presence of SGK1.1 (K220A) (Figure 6D). These findings demonstrate that the activity of the kinase disrupts the association between Nedd4-2 and the channel. Importantly, our data additionally show that the association between the channel and the ubiquitin ligase was significantly diminished in the presence of either of the epileptogenic Kv7.2 mutations (Figures 6C,D).

\section{DISCUSSION}

The results presented here, in agreement with our previously published study (Miranda et al., 2013), demonstrate that the neuronal isoform SGK1.1 selectively upregulates activity of heteromeric Kv7.2/3 and Kv7.3/5 channels, while it fails to regulate or even decreases the activity of homomeric channels formed by Kv7.2 or 7.3 (Miranda et al., 2013), Kv7.4 or 7.5. In addition, SGK1.1 was not able to modulate the current elicited by Kv7.1/KCNE1, a subunit combination that generates the major repolarizing cardiac current, $\mathrm{I}_{\mathrm{Ks}}$. In contrast, the 

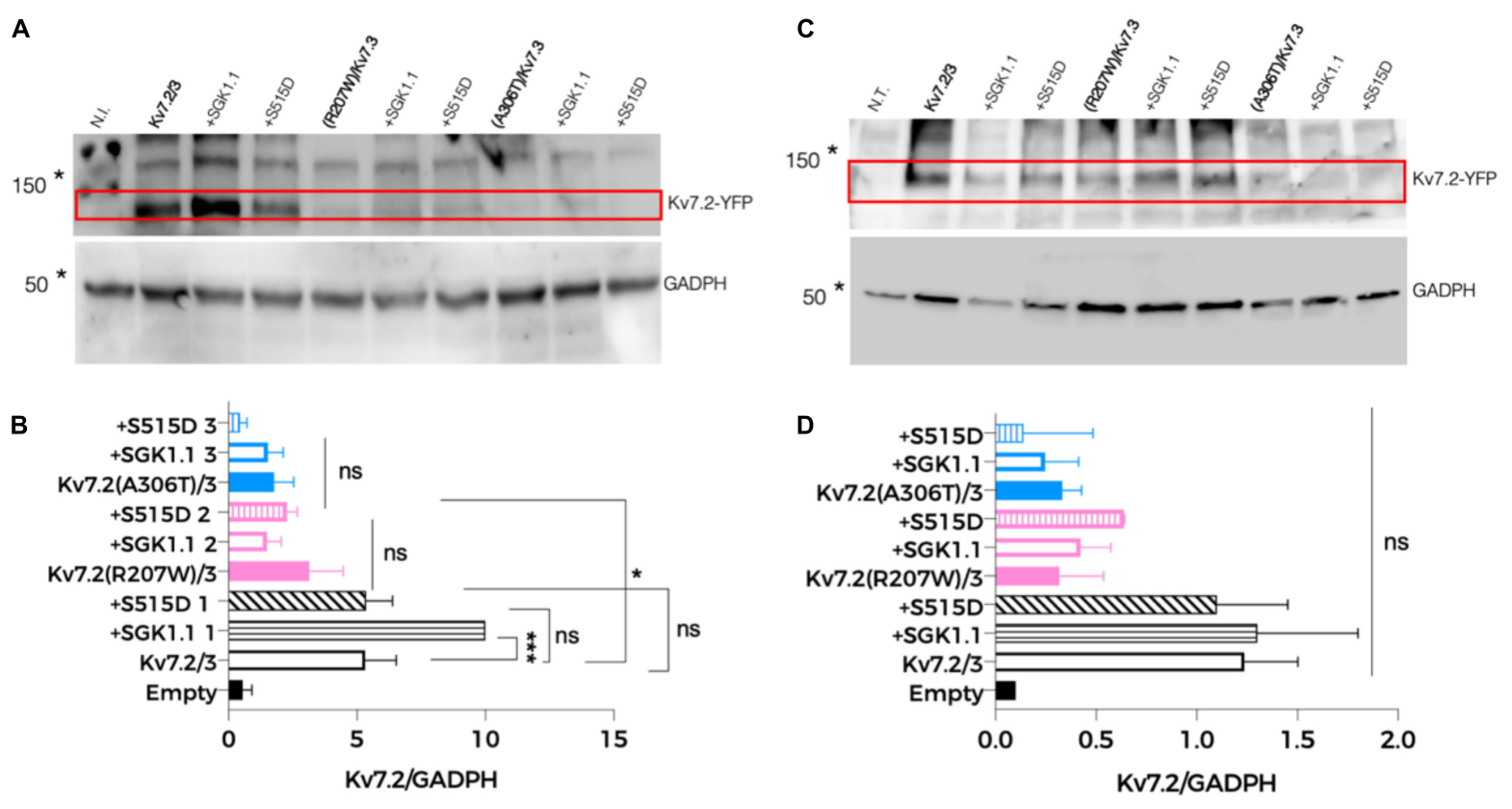

E

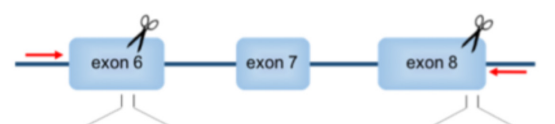

$\frac{\text { CTTCTTGAAAGTGATCGGAAAGG }}{\text { gRNA }} \frac{\text { GTCCTGGACTACATTAATGGTGG }}{\text { gRNA }}$

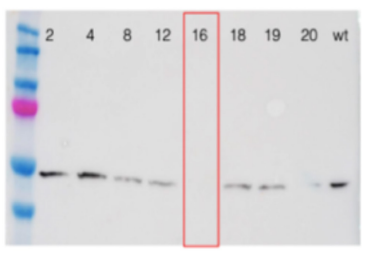

F

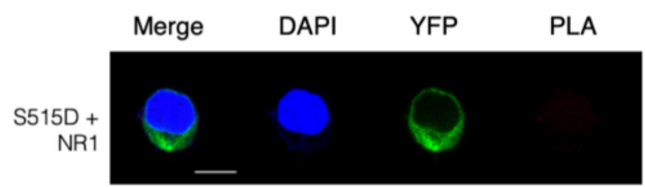

G
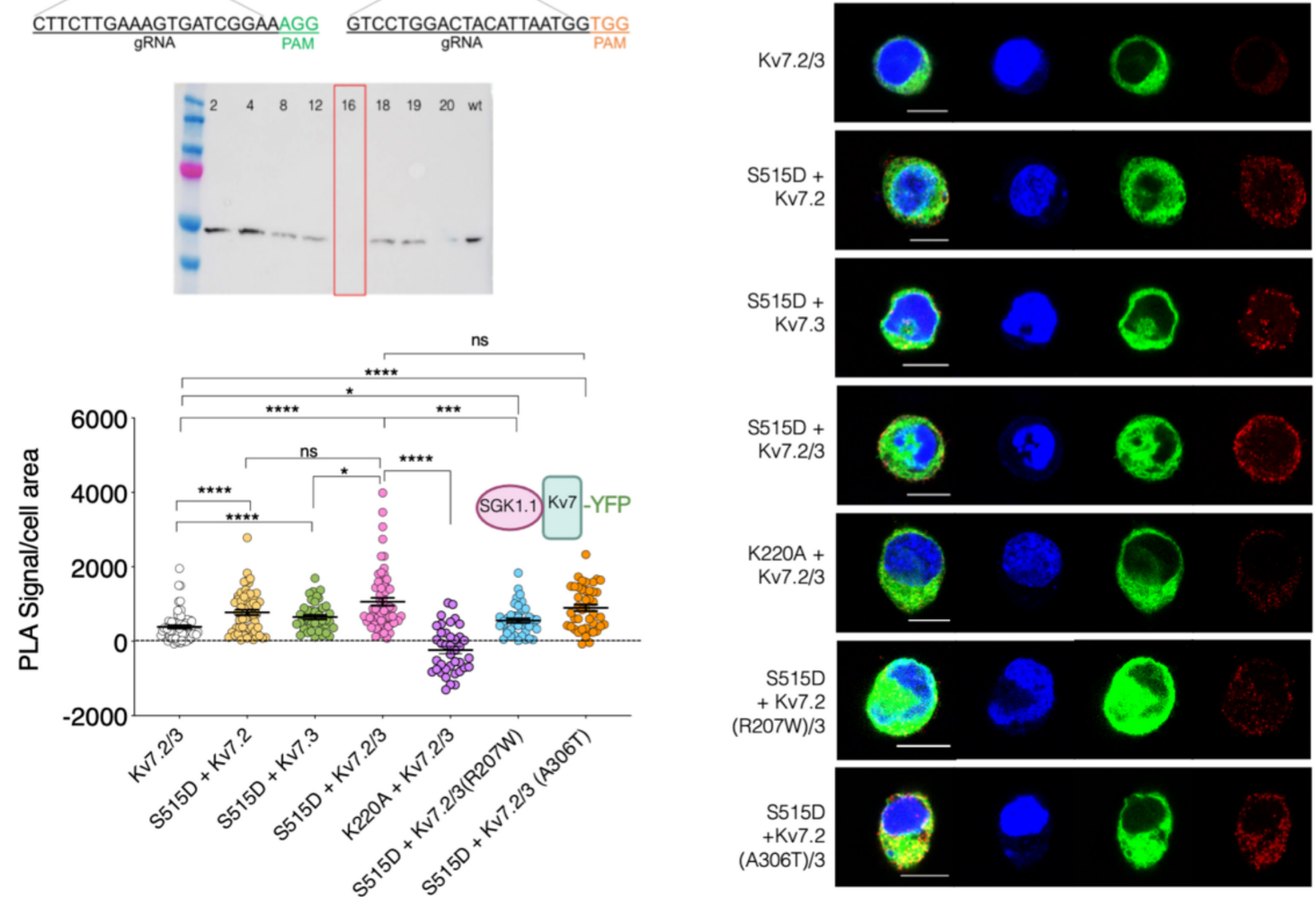

FIGURE 5 | Association between SGK1.1 (S515D) and heteromeric channel Kv7.2/3 is reduced in presence of epilepsy mutant Kv7.2 (R207W) but not Kv7.2 (A306T). (A) Representative immunoblot showing expression levels of Kv7.2 fused to YFP (top panel, $110 \mathrm{KDa}$ ) and GADPH (bottom panel, $50 \mathrm{KDa}$ ) from oocytes injected with the indicated constructs; N.I., water-injected oocytes. Asterisks denote migration of the indicated molecular mass marker. (B) Quantitative analysis of KV7.2 expression levels in Xenopus oocytes. Values are mean \pm SEM from at least three independent experiments (ANOVA Sidak's test for multiple comparisons; ns, 
FIGURE 5 | not significant; ${ }^{*} p<0.05 ;{ }^{\star \star \star} p<0.0005$ ). (C) Representative western blot showing expression levels of Kv7.2 fused to YFP (top panel, 110 KDa approximately) and GADPH (bottom panel, $50 \mathrm{KDa}$ ) from N2a Sgk1-KO cells transfected with the indicated constructs; N.T., non-transfected cells. Asterisks denote migration of the indicated molecular mass marker. (D) Quantitative analysis of Kv7.2 expression levels in N2a cells from two independent replicates (ANOVA Sidak's test for multiple comparisons; ns, not significant). (E) Top, schematic representation of the CRISPR/Cas9 strategy to knockout the Sgk1 gene. Insets indicate the sequence and hybridization sites for the guides, and PAM sequences (NGG) required for Cas9 to cut the DNA at the end of each targeted exon. Screening PCR primers are indicated as red arrows. Bottom, western blot detecting the presence of SGK1 protein expression in WT N2a cells or in selected single cell clones. Clone 16 was selected and used as Sgk1 knockout in this study. (F) PLA was performed on N2a Sgk1-KO cells transfected with the indicated constructs. Cells transfected with SGK1.1 (S515D) and NR1 were used as negative control (see F and dotted line at 0 in G). Bars correspond to $10 \mu M$. (G) Quantification of PLA positive signals for each condition (ANOVA Kruskal Wallis test, ns, not significant; ${ }^{\star} p<0.05 ;{ }^{\star \star *} p<0.0005 ;{ }^{* \star \star} p<0.0001$ ). Each dot represents an individual cell from at least three independent experiments.

ubiquitous isoform SGK1 has been described to increase $\mathrm{I}_{\mathrm{Ks}}$ in Xenopus laevis oocytes in a Nedd4-2 dependent manner (Seebohm et al., 2008). This leads us to hypothesize that the differential $\mathrm{NH}_{2}$-terminal domain present in SGK1.1 and its ability to interact with $\mathrm{PIP}_{2}$ could be responsible for the selectivity of the neuronal isoform of the kinase toward heteromeric channels underlying the M-current. Similar to Kv7.2 and Kv7.3, Kv7.5 is expressed in the brain and yields currents that form heteromeric channels with Kv7.3 and are inhibited by M1 muscarinic receptor activation (Schroeder et al., 2000). Our results demonstrate the ability of SGK1.1 to increase Kv7.3/5-elicited M-currents, an effect that could contribute to the anticonvulsant activity of the kinase (Miranda et al., 2013; Armas-Capote et al., 2020). In fact, some studies demonstrating activation of Kv7.3/5 by retigabine suggested it might constitute a molecular target for this agent along with Kv7.2/3 (Wickenden et al., 2001). In addition, SGK1.1 is mainly expressed in pyramidal neurons (Wesch et al., 2010; MartinBatista et al., 2021). Altogether, these data indicate that SGK1.1 activation could be a promising therapeutic target for epilepsy, as it shares the mechanism of action of retigabine without substantial risk of activating potassium channels expressed outside neuronal tissue.

As previously mentioned, among the different causes for epilepsy, genetic mutations affecting the functionality of Kv7.2-5 channels have been described in diverse forms of the disease. We have assessed the power of SGK1.1 to upregulate the M-current in the presence of two epilepsy-associated Kv7.2 mutations. Our results suggest that constitutive activation of the kinase, which does not have an additional effect on WT Kv7.2/3generated $\mathrm{M}$-current, is able to significantly restore activity of loss-of-function epileptogenic mutations Kv7.2 (R207W) and Kv7.2 (A306T). Mutation R207W, which results in BFNC and myokymia (Dedek et al., 2001), neutralizes a charged amino acid residue within the S4 segment, affecting the voltage sensor domain and slowing voltage-sensor dependent activation. Kv7.2 (A306T) is located in the S6 segment (pore domain) and also associated with BFNC. In this study, we show that both mutations also result in a reduction in protein expression levels, at least in heterologous expression systems. Thus, our findings might contribute to understand the phenotype observed in patients carrying these mutations because, other than being affected by the intrinsic malfunction of the mutated channel, we can now suggest that the expression of the protein and the ability of wild type SGK1.1 to functionally upregulate it are disturbed. Therefore, activated SGK1.1 could constitute a strategy to increase the $\mathrm{M}$-current in the presence of mutations that diminish this potassium current. Whether or not pharmacological activation of SGK1.1 may provide a useful therapeutic approach should be further addressed.

As part of our interest in understanding the effect of epileptogenic Kv7.2 mutations on the SGK1.1 mechanism of action, we evaluated the physical proximity between the kinase and the channel by PLA. Our results demonstrate that the association between SGK1.1 and heteromeric Kv7.2/3 was significantly higher than that observed in the presence of homomeric Kv7.3 channels, in agreement with the functional data, which indicate that the SGK1.1 effect requires the heteromeric co-assembly of Kv7.2/3 (Miranda et al., 2013) or Kv7.3/5 channels. The fact that SGK1.1 still associates with, but fails to regulate, homomeric channels supports the hypothesis that the mechanism controlling their plasma membrane levels is different from the one regulating heteromeric combinations of Kv7 subunits. Association of SGK1.1 with the heteromeric channel was significantly impaired in the inactive mutant SGK1.1 (K220A), suggesting that conformational changes associated with the catalytic activity of the kinase are crucial for its interaction with the channel. In addition, we were able to detect association of Nedd4-2 with SGK1.1 and, most importantly, to Kv7 channels in a SGK1.1-dependent manner. As we have previously demonstrated, SGK1.1 enhances the levels of phosphorylation of Nedd4-2 at residue S448 (Armas-Capote et al., 2020), similarly to SGK1, a process that results in the repression of Nedd42 ubiquitylation activity (Debonneville et al., 2001). This may prevent M-channel degradation, stabilizing it in the membrane. Consistently, we found that constitutively active, but not the kinase-dead mutant of SGK1.1 is able to displace Nedd4-2 from its interaction with the M-channel.

Co-expression of SGK1.1 with Kv7.2 epilepsy mutants produced divergent results. Both mutants were still able to closely associate with SGK1.1, even though they are expressed at significantly reduced levels. Therefore, it appears that overall Kv7 subunit abundance is not a predictor of SGK1.1 association, which may be compartment-specific and depend on the relative abundance of the channel in the plasma membrane vs. intracellular compartments. In contrast, association between Nedd4-2 and Kv7 was significantly reduced in the presence of either epilepsy mutation. Given that both mutations affect different functional regions of the channel, it is unlikely that they directly control the ability of Kv7.2 to interact with Nedd42. We speculate that their presence indirectly alters the ability 


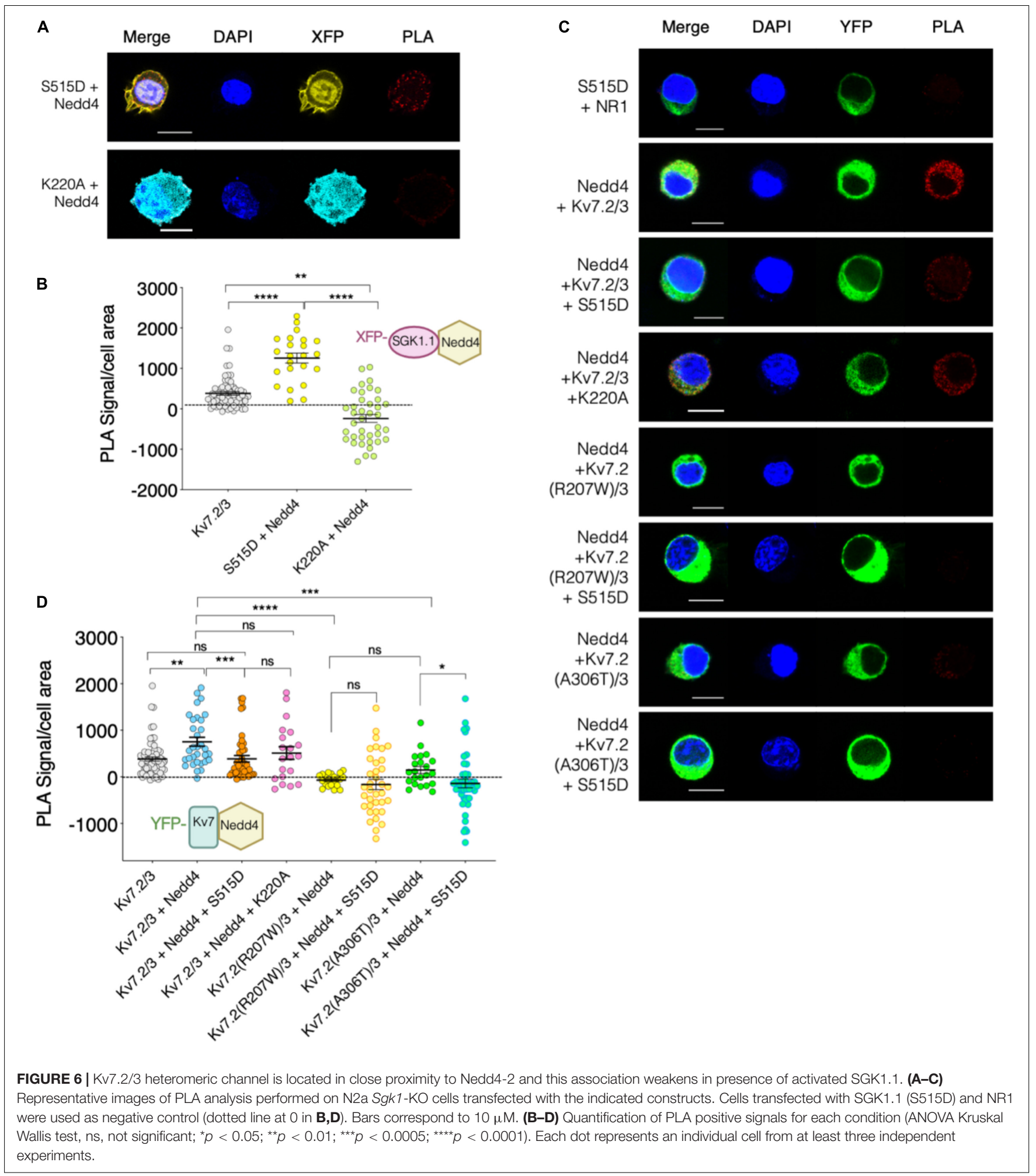

of Nedd4-2 to interact with Kv7.2, possibly by altering its subcellular localization and/or expression levels. In summary, it seems clear that SGK1.1 can still interact with the M-channel independently of the presence of Nedd4-2 in the complex and regardless of mutations altering Kv7.2 activity/expression. Thus, it is tempting to speculate that activation of the kinase might be up-regulating the $\mathrm{M}$-current through an alternative mechanism in addition to the Nedd4-2 pathway. This mechanism would be more prominent in the case of the epileptogenic mutations, since these mutants lack the interaction with Nedd4-2 and 
therefore are not subject to the same regulatory pathway as the WT channel. One possibility for such alternative pathway of regulation is phosphorylation. As has been proposed for SGK1 (Bongiorno et al., 2011), SGK1.1 could interact directly with the RXRXXS/T consensus motif in the subunits of ion channels, additionally to the indirect association through the PY motif present in Nedd4-2. The Kv7 subunit sequence shows interaction sites for phosphorylation on serine, threonine and tyrosine residues (Ismailov and Benos, 1995). Even though the consensus phosphorylation motif of SGK1 has only been described in Kv7.4 (Seebohm et al., 2005), the cytoplasmic $\mathrm{N}$-terminal domain in Kv7.2 contains a consensus site for cAMP-dependent phosphorylation by PKA that is required for its stimulation by cAMP (Schroeder et al., 1998). PKA, whose consensus sequence overlaps with that of SGK1, not only phosphorylates Kv7 but also Nedd4-2 at the same sites as does SGK1. Hence it would be possible that SGK1.1 phosphorylates the channel likewise (Snyder et al., 2004). In addition, mass spectrometry studies have revealed different phosphorylation sites in the S4-S5 loop of Kv7.2/3 within a sequence highly conserved among Kv7 family members (Surti et al., 2005). Whether SGK1.1 is able to directly phosphorylate Kv7 channel subunits remains unexplored. Alternatively, the kinase might be indirectly regulating channel trafficking. For instance, it has been previously demonstrated that the ubiquitous isoform SGK1 regulates Kv7.1/KCNE1 heteromer trafficking via Rab11-mediated recycling (Seebohm et al., 2008). It is clear from our data that the interaction between SGK1.1, Nedd42 , and Kv7 a subunits is complex and depends not only on total protein abundance, but also on enzymatic activity, subcellular localization and the presence or absence of certain epilepsy mutations.

In summary, our study demonstrates that the neuronal isoform SGK1.1 selectively upregulates Kv7 subunit heteromers underlying the M-current and that activation of this kinase may provide a therapeutic target for treating epilepsy, particularly in patients carrying specific Kv7.2 epileptogenic mutations.

\section{DATA AVAILABILITY STATEMENT}

The raw data supporting the conclusions of this article will be made available by the authors, without undue reservation.

\section{REFERENCES}

Armas-Capote, N., Maglio, L. E., Pérez-Atencio, L., Martin-Batista, E., Reboreda, A., Barios, J. A., et al. (2020). SGK1.1 Reduces Kainic Acid-Induced Seizure Severity and Leads to Rapid Termination of Seizures. Cereb. Cortex 30, 31843197. doi: 10.1093/cercor/bhz302

Arteaga, M. F., Coric, T., Straub, C., and Canessa, C. M. (2008). A brain-specific SGK1 splice isoform regulates expression of ASIC1 in neurons. Proc. Natl. Acad. Sci. U. S. A. 105, 4459-4464. doi: 10.1073/pnas.0800958105

Bongiorno, D., Schuetz, F., Poronnik, P., and Adams, D. J. (2011). Regulation of voltage-gated ion channels in excitable cells by the ubiquitin ligases Nedd 4 and Nedd4-2. Channels 5, 79-88. doi: 10.4161/chan.5.1.13967

Brown, D. A., and Adams, P. (1980). Summary for Policymakers in Intergovernmental Panel on Climate Change, Vol. 283. Cambridge: Cambridge University Press, 1-30.

\section{ETHICS STATEMENT}

The animal study was reviewed and approved by the Universidad de La Laguna Ethics Committee.

\section{AUTHOR CONTRIBUTIONS}

EM-B: conceptualization, investigation, formal analysis, writingoriginal draft, writing-review and editing, and visualization. RM, BR-P, and DB-M: conceptualization, investigation, formal analysis, and writing-review. DA: conceptualization, formal analysis, writing-original draft, writing-review and editing, and visualization. GA: conceptualization, formal analysis, writingreview and editing, funding acquisition, supervision, and project administration. TG: conceptualization, formal analysis, writingoriginal draft, writing-review and editing, funding acquisition, supervision, visualization, project administration, and validation. All authors contributed to the article and approved the submitted version.

\section{FUNDING}

This work was supported by the Ministerio de Ciencia e Innovación - MICINN-, Spain (grant nos. BFU2015-66490R, RTI2018-098768-B-I00, BFU2015-70067-REDC to TG; F.P.I. predoctoral Fellowship BES-2016-077337 to EM-B); the European Research Council (ERC) under the European Union Horizon 2020 research and innovation program (grant agreement 648936); the Spanish Cabildo Insular de Tenerife Program Agustin de Betancourt (grant no. AB2019/007 to BR-P) and the United States National Institutes of Health, National Institute of General Medical Sciences (grant no. GM130377 to GA).

\section{SUPPLEMENTARY MATERIAL}

The Supplementary Material for this article can be found online at: https://www.frontiersin.org/articles/10.3389/fnmol. 2021.798261/full\#supplementary-material

Clark, S., Antell, A., and Kaufman, K. (2015). New antiepileptic medication linked to blue discoloration of the skin and eyes. Ther. Adv. Drug Saf. 6, 15-19. doi: $10.1177 / 2042098614560736$

Constanti, A., and Brown, D. A. (1981). M - currents in voltage - clamped mammalin sympathetic neurones. Neurosci. Lett. 24, 289-294.

Debonneville, C., Flores, S. Y., Kamynina, E., Plant, P. J., Tauxe, C., Thomas, M. A., et al. (2001). Phosphorylation of Nedd4-2 by Sgk1 regulates epithelial $\mathrm{Na}(+)$ channel cell surface expression. EMBO J. 20, 7052-7059. doi: 10.1093/emboj/ 20.24.7052

Dedek, K., Kunath, B., Kananura, C., Reuner, U., Jentsch, T. J., and Steinlein, O. K. (2001). Myokymia and neonatal epilepsy caused by a mutation in the voltage sensor of the KCNQ2 K+ channel. Proc. Natl. Acad. Sci. U. S. A. 98, 12272-12277. doi: 10.1073/pnas.211431298

Ekberg, J., Schuetz, F., Boase, N. A., Conroy, S. J., Manning, J., Kumar, S., et al. (2007). Regulation of the voltage-gated K+ channels KCNQ2/3 and KCNQ3/5 
by ubiquitination: Novel role for Nedd4-2. J. Biol. Chem. 282, 12135-12142. doi: 10.1074/jbc.M609385200

Gomez-Posada, J. C., Aivar, P., Alberdi, A., Alaimo, A., Etxeberria, A., FernandezOrth, J., et al. (2011). Kv7 channels can function without constitutive calmodulin tethering. PLoS One 6:e25508. doi: 10.1371/journal.pone.0025508

Ismailov, I. I., and Benos, D. J. (1995). Effects of phosphorylation on ion channel function. Kidney Int. 48, 1167-1179. doi: 10.1038/ki.1995.400

Kubisch, C., Schroeder, B. C., Friedrich, T., Lütjohann, B., El-Amraoui, A., and Marlin, S. (1999). KCNQ4, a novel potassium channel expressed in sensory outer hair cells, is mutated in dominant deafness. Cell 96, 437-446. doi: 10.1016/ S0092-8674(00)80556-5

Lerche, C., Scherer, C. R., Seebohm, G., Derst, C., Wei, A. D., Busch, A. E., et al. (2000). Molecular cloning and functional expression of KCNQ5, a potassium channel subunit that may contribute to neuronal M-current diversity. J. Biol. Chem. 275, 22395-22400. doi: 10.1074/jbc.M002378200

Luo, J.-H., Fu, Z. Y., Losi, G., Kim, B. G., Prybylowski, K., Vissel, B., et al. (2002). Functional expression of distinct NMDA channel subunits tagged with green fluorescent protein in hippocampal neurons in culture. Neuropharmacology 42 , 306-318. doi: 10.1016/S0028-3908(01)00188-5

Manville, R. W., Papanikolaou, M., and Abbott, G. W. (2018). Direct neurotransmitter activation of voltage-gated potassium channels. Nat. Commun. 9:1847. doi: 10.1038/s41467-018-04266-w

Martin-Batista, E., Maglio, L. E., Armas-Capote, N., Hernández, G., Alvarez de la Rosa, D., and Giraldez, T. (2021). SGK1.1 limits brain damage after status epilepticus through $\mathrm{M}$ current-dependent and independent mechanisms. Neurobiol. Dis. 153:105317. doi: 10.1016/j.nbd.2021.105317

Miceli, F., Soldovieri, M. V., Joshi, N., Weckhuysen, S., Cooper, E., and Taglialatela, M. (2010). KCNQ2-Related Disorders. GeneReviews 2010:27.

Michel, M. C., Radziszewski, P., Falconer, C., Marschall-Kehrel, D., and Blot, K. (2012). Unexpected frequent hepatotoxicity of a prescription drug, flupirtine, marketed for about 30 years. Br. J. Clin. Pharmacol. 73, 821-825. doi: 10.1111/j. 1365-2125.2011.04138.x

Miranda, P., Cadaveira-Mosquera, A., González-Montelongo, R., Villarroel, A., and González-Hernández, T. (2013). The Neuronal Serum- and Glucaocorticoid-Regulated Kinase 1.1 Reduces Neuronal Excitability and Protects against Seizures through Upregulation of the M-Current. J. Neurosci. 33, 2684-2696. doi: 10.1523/JNEUROSCI.3442-12.2013

Nappi, P., Miceli, F., Soldovieri, M. V., Ambrosino, P., Barrese, V., and Taglialatela, M. (2020). Epileptic channelopathies caused by neuronal Kv7 (KCNQ) channel dysfunction. Pflugers Arch. Eur. J. Physiol. 472, 881-898. doi: 10.1007/s00424020-02404-2

Oliveros, J. C., Franch, M., Tabas-Madrid, D., San-León, D., Montoliu, L., Cubas, P., et al. (2016). Breaking-Cas-interactive design of guide RNAs for CRISPRCas experiments for ENSEMBL genomes. Nucleic Acids Res. 44, W267-W271. doi: $10.1093 / \mathrm{nar} / \mathrm{gkw} 407$

Oyrer, J., Maljevic, S., Scheffer, I. E., Berkovic, S. F., Petrou, S., and Reid, C. A. (2018). Ion channels in genetic epilepsy: From genes and mechanisms to disease-targeted therapies. Pharmacol. Rev. 70, 142-173. doi: 10.1124/pr.117. 014456

Plouin, P., and Anderson, V. (2005). Epileptic Syndromes in Infancy, Childhood and Adolescence, 4th Edn. Montrouge: John Libbey Eurotext.

Ran, F. A., Hsu, P. D., Wright, J., Agarwala, V., Scott, D. A., and Zhang, F. (2013). Genome engineering using the CRISPR-Cas9 system. Nat. Protoc. 8, 2281-2308. doi: 10.1038/nprot.2013.143

Ronen, G. M., Rosales, T. O., Connolly, M., Anderson, V. E., and Leppert, M. (1993). Seizure characteristics in chromosome 20 benign familial neonatal convulsions. Neurology 43, 1355-1360.

Schroeder, B. C., Hechenberger, M., Weinreich, F., Kubisch, C., and Jentsch, T. J. (2000). KCNQ5, a Novel Potassium Channel Broadly Expressed in Brain, Mediates M-type Currents. J. Biol. Chem. 275, 24089-24095. doi: 10.1074/jbc. M003245200

Schroeder, B. C., Kubisch, C., Stein, V., and Jentsch, T. J. (1998). Moderate loss of function of cyclic-AMP-modulated KCNQ2/KCNQ3 K+ channels causes epilepsy. Nature 396, 687-690. doi: 10.1038/25367

Seebohm, G., Strutz-Seebohm, N., Baltaev, R., Korniychuk, G., Knirsch, M., Engel, J., et al. (2005). Regulation of KCNQ4 Potassium Channel Prepulse Dependence and Current Amplitude by SGK1 in Xenopus oocytes. Cell. Physiol. Biochem. 16, 255-262. doi: 10.1159/000089851

Seebohm, G., Strutz-Seebohm, N., Ureche, O. N., Henrion, U., Baltaev, R., Mack, A. F., et al. (2008). Long QT Syndrome-Associated Mutations in KCNQ1 and
KCNE1 Subunits Disrupt Normal Endosomal Recycling of I Ks Channels. Circ. Res. 103, 1451-1457. doi: 10.1161/CIRCRESAHA.108.177360

Shah, M. M., Mistry, M., Marsh, S. J., Brown, D. A., and Delmas, P. (2002). Molecular correlates of the M-current in cultured rat hippocampal neurons. J. Physiol. 544, 29-37. doi: 10.1113/jphysiol.2002.028571

Singh, N. A., Charlier, C., Stauffer, D., DuPont, B. R., Leach, R. J., Melis, R., et al. (1998). A novel potassium channel gene, KCNQ2, is mutated in an inherited epilepsy of newborns. Nat. Genet. 18, 25-29. doi: 10.1038/ ng0198-25

Singh, N. A., Westenskow, P., Charlier, C., Pappas, C., Leslie, J., Dillon, J., et al. (2003). KCNQ2 and KCNQ3 potassium channel genes in benign familial neonatal convulsions: Expansion of the functional and mutation spectrum. Brain 126, 2726-2737. doi: 10.1093/brain/awg286

Snyder, P. M., Olson, D. R., Kabra, R., Zhou, R., and Steines, J. C. (2004). cAMP and serum and glucocorticoid-inducible kinase (SGK) regulate the epithelial $\mathrm{Na}+$ channel through convergent phosphorylation of Nedd4-2. J. Biol. Chem. 279, 45753-45758. doi: 10.1074/jbc.M407858200

Soldovieri, M. V., Boutry-Kryza, N., Milh, M., Doummar, D., Heron, B., Bourel, E., et al. (2014). Novel KCNQ2 and KCNQ3 mutations in a large cohort of families with benign neonatal epilepsy: First evidence for an altered channel regulation by syntaxin-1A. Hum. Mutat. 35, 356-367. doi: 10.1002/humu.2 2500

Surti, T. S., Huang, L., Jan, Y. N., Jan, L. Y., and Cooper, E. C. (2005). Identification by mass spectrometry and functional characterization of two phosphorylation sites of KCNQ2/KCNQ3 channels. Proc. Natl. Acad. Sci. U. S. A. 102, 1782817833. doi: 10.1073/pnas.0509122102

Surur, A. S., Bock, C., Beirow, K., Wurm, K., Schulig, L., Kindermann, M. K., et al. (2019). Flupirtine and retigabine as templates for ligand-based drug design of K V 7.2/3 activators. Org. Biomol. Chem. 17, 4512-4522. doi: 10.1039/c9ob0 $0511 \mathrm{k}$

Wang, H. S., and McKinnon, D. (1995). Potassium currents in rat prevertebral and paravertebral sympathetic neurones: control of firing properties. J. Physiol. 485, 319-335. doi: 10.1113/jphysiol.1995.sp020732

Wang, H. S., Pan, Z., Shi, W., Brown, B. S., Wymore, R. S., Cohen, I. S., et al. (1998). KCNQ2 and KCNQ3 potassium channel subunits: molecular correlates of the M-channel. Science 282, 1890-1893. doi: 10.1126/science.282.5395.1890

Wesch, D., Miranda, P., Afonso-Oramas, D., Althaus, M., Castro-Hernández, J., Dominguez, et al. (2010). The neuronal-specific SGK1 . 1 kinase regulates $\delta$-epithelial $\mathrm{Na}+$ channel independently of PY motifs and couples it to phospholipase C signaling. Am. J. Cell Physiol. 299, 779-790. doi: 10.1152/ ajpcell.00184.2010

Wickenden, A. D., Roeloffs, R., McNaughton-Smith, G., and Rigdon, G. C. (2004). KCNQ potassium channels: Drug targets for the treatment pf epilepsy and pain. Expert Opin. Ther. Pat. 14, 457-469. doi: 10.1517/13543776.14.4.457

Wickenden, A. D., Zou, A., Wagoner, P. K., and Jegla, T. (2001). Characterization of KCNQ5/Q3 potassium channels expressed in mammalian cells. $\mathrm{Br} . \mathrm{J}$. Pharmacol. 132, 381-384. doi: 10.1038/sj.bjp.0703861

Wulff, H., Castle, N. A., and Pardo, L. A. (2009). Voltage-gated potassium channels as therapeutic targets. Nat. Rev. Drug Discov. 8, 982-1001. doi: 10.1038/nrd2983

Xiong, Q., Sun, H., Zhang, Y., Nan, F., and Li, M. (2008). Combinatorial augmentation of voltage-gated KCNQ potassium channels by chemical openers. Proc. Natl. Acad. Sci. U. S. A. 105, 3128-3133. doi: 10.1073/pnas.0712256105

Conflict of Interest: The authors declare that the research was conducted in the absence of any commercial or financial relationships that could be construed as a potential conflict of interest.

Publisher's Note: All claims expressed in this article are solely those of the authors and do not necessarily represent those of their affiliated organizations, or those of the publisher, the editors and the reviewers. Any product that may be evaluated in this article, or claim that may be made by its manufacturer, is not guaranteed or endorsed by the publisher.

Copyright (c) 2021 Martin-Batista, Manville, Rivero-Pérez, Bartolomé-Martín, Alvarez de la Rosa, Abbott and Giraldez. This is an open-access article distributed under the terms of the Creative Commons Attribution License (CC BY). The use, distribution or reproduction in other forums is permitted, provided the original author(s) and the copyright owner(s) are credited and that the original publication in this journal is cited, in accordance with accepted academic practice. No use, distribution or reproduction is permitted which does not comply with these terms. 